\title{
Selective versus Non-selective Culture Medium for Group B Streptococcus Detection in Pregnancies Complicated by Preterm Labor or Preterm-Premature Rupture of Membranes
}

\author{
Marcelo Luís Nomura ${ }^{1}$, Renato Passini Júnior ${ }^{1}$ \\ Ulysses Moraes Oliveira²
}

\author{
${ }^{1}$ Department of Obstetrics and Gynecology; ${ }^{2}$ Department of \\ Clinical Pathology; Medical School, Campinas State University \\ (UNICAMP), São Paulo State, Brazil
}

\begin{abstract}
The objective of this study was to identify group B streptococcus (GBS) colonization rates and compare detection efficiency of selective versus non-selective culture media and anorectal versus vaginal cultures in women with preterm labor and preterm-premature rupture of membranes (PROM). A prospective cohort study of 203 women was performed. Two vaginal and two anorectal samples from each woman were collected using sterile swabs. Two swabs (one anorectal and one vaginal) were placed separately in Stuart transport media and cultured in blood-agar plates for 48 hours; the other two swabs were inoculated separately in Todd-Hewitt selective media for $\mathbf{2 4}$ hours and then subcultured in blood-agar plates. Final GBS identification was made by the CAMP test. A hundred thrity-two cultures out of 812 were positive. The maternal colonization rate was $27.6 \%$. Colonization rates were $30 \%$ for preterm PROM and $25.2 \%$ for preterm labor. Todd-Hewitt selective medium detected $87.5 \%$ and non-selective medium $60.7 \%$ GBS-positive women. Vaginal samples and anorectal samples had the same detection rate of $80.3 \%$. Anorectal selective cultures detected $75 \%$ of carriers; $39 \%$ of GBS-positive women were detected only in selective medium. A combined vaginal-anorectal selective culture is appropriate for GBS screening in this population, minimizing laboratory costs.
\end{abstract}

Key Words: Streptococcus agalactiae, culture media, premature rupture of membranes, preterm labor.

Streptococcus agalactiae, or group B streptococcus (GBS), is an important infectious agent in perinatology; it is the predominant bacteria in early-onset neonatal sepsis [1,2]. GBS can be acquired during labor or in utero by transmission from maternal-vaginal or anorectal-colonized mucosa. Prematurity is also a risk factor, and mortality is higher in preterm than in term newborns [3].

Reported maternal colonization rates are quite variable, but generally range from $20-30 \%$ [4,5]. The differences in colonization rates depend on the particular population and especially on the laboratory methods used to identify GBS. Brazilian data are scarce [6,7], but are in general agreement with literature reports from other countries.

Since maternal colonization at delivery is the main risk factor for neonatal disease [4], microbiological techniques must be designed in order to maximize detection rates. The use of selective media containing antibiotics is reported to be both sensitive and also the most adequate method for detection [5]. The anatomic site of sampling is also important, and anorectal and vaginal cultures are recommended for detection in pregnant women [8].

Received on 12 April 2006; revised 26 July 2006.

Address for correspondence: Dr. Marcelo Luís Nomura. Rua Alexander Fleming, 101, Cidade Universitária Zeferino Vaz - Área de Obstetrícia - Centro de Atenção Integral a Saúde da Mulher Universidade Estadual de Campinas-Campinas, São Paulo, Zip code: 13084-881-Brazil..E-mail:mlnomura@unicamp.br.

The Brazilian Journal of Infectious Diseases 2006;10(4):247-250. (C) 2006 by The Brazilian Journal of Infectious Diseases and Contexto Publishing. All rights reserved.
We analyzed culture methods for the detection of GBS in pregnancies complicated by preterm labor (PTL) or preterm premature rupture of membranes (PPROM), comparing ToddHewitt selective medium with non-selective medium and anorectal with vaginal samples.

\section{Material and Methods}

During the 12-month period from February 2003 to January 2004, all pregnant women (total 203) with preterm labor or preterm premature rupture of membranes attended at the Maternity Ward of the Campinas State University, located in Campinas, in the state of São Paulo, Brazil were enrolled and included in the study. All participating women signed an informed consent. Socio-demographic, clinical and obstetrical data were collected at the time of admission and retrospectively from patient's charts.

Samples were collected from the proximal third of the vaginal introitus and from inside the anus through the anal sphincter. Two vaginal and two anorectal swabs were obtained from each woman. Two swabs (one vaginal and one anorectal) were separately placed in two tubes containing Stuart transport media and then cultured in non-selective $5 \%$ bloodagar plates for 48 hours. The other two swabs were immediately inoculated into two tubes containing Todd-Hewitt selective broth medium (manufactured by PROBAC, Brazil), enriched with gentamicin and nalidixic acid, incubated for 24 hours, and then subcultured for 24 hours in non-selective $5 \%$ bloodagar plates. The final identification of GBS in all samples was made by the CAMP test, which is based on the production of an arrow-shaped hemolysis zone in conjunction with a Staphylococcus aureus colony in a blood-agar plate. Four 
types of culture were then identified: selective anorectal, nonselective anorectal, selective vaginal and non-selective vaginal. A woman was considered colonized if any of the four cultures were positive.

Detection rates for each type of culture medium and each sampling site were calculated. Chi-square or Fischer's exact test were used to detect significant differences, and a P value $<0.05$ was considered statistically significant.

\section{Results}

One hundred thirty two (16.2\%) GBS-positive cultures were obtained from the 812 specimens collected from the 203 pregnant women. Fifty-six women (27.6\%) had at least one of the cultures positive. Table 1 shows the clinical and obstetric characteristics of the women enrolled in the study. White race and low education level were significantly associated with colonization.

Colonization rates for women in preterm labor were $25.2 \%$, and for women with preterm premature rupture of membranes it was 30\%. Sixteen women (7.8\%) had all of the four cultures positive. Selective anorectal cultures were positive in 42 patients (20.7\%) and selective vaginal cultures were positive in 38 (18.7\%). Non-selective anorectal cultures were positive in 24 patients (11.8\%) and non-selective vaginal cultures in 28 (13.8\%). Table 2 shows the comparison of the selective versus non-selective cultures. There was discordance in the results for 29 women. Twenty-two (39)\% of the colonized women were detected only in the selective-media cultures.

Table 3 shows culture results by site of sampling. Discordance between results was observed in 22 women. However, the number of colonized women detected by each of the cultures was the same (45 each, or $80.3 \%$ of all colonized women). However, these 22 women were not the same in each group.

Selective media detected $87.5 \%$ of the 56 colonized women, compared to $60.7 \%$ detected by non-selective cultures. There was no difference in the detection rate between anorectal and vaginal samples (80.3\%).

\section{Discussion}

Screening of group B streptococcus colonization is recommended for all pregnant women between 35 and 37 weeks, and in situations of risk of preterm delivery, which are preterm labor and preterm premature rupture of membranes [5]. Antibiotic prophylaxis of colonized women, during labor, greatly reduces the risk of neonatal disease [9].

It has been reported that GBS isolation is $20 \%$ to $40 \%$ greater when combined vaginal and anorectal cultures are collected [8,10-12]. A significant proportion of women were found to have only one of these sites colonized; this proportion was $18 \%$ to $24 \%$ higher in anorectal compared to vaginal samples $[11,12]$. In an analysis of 651 specimens, the combination of anorectal and vaginal cultures reduced the number of false-negative results, allowing detection of 97.8\% of GBS carriers [13]. Since there are no published data comparing the risk of neonatal disease by colonized anatomic site, it is recommended that both vagina and rectum be cultured [5].

In our study, 11 patients were detected only in vaginal and another 11 only in anorectal cultures; the number of carriers detected would have been the same if only one of these sites was sampled. However, anorectal-selective medium detected $75 \%$ of GBS carriers.

Todd-Hewitt selective medium enriched with gentamicin and nalidixic acid inhibits growth of Gram-negative bacteria, and it has greater sensitivity when compared to other nonselective media, such as blood or Granada agar [14]. Twentytwo cases, or 39\% of all colonized women in this study, were detected only in the samples incubated in Todd-Hewitt medium. The proportion of colonized women detected by the selective medium was $87.5 \%$, and 15 affected patients would not have been detected if only non-selective medium had been used; the detection rate would have fallen $30 \%$. This finding supports the current view that selective medium is fundamental to maximize detection rates and should be employed by laboratories involved in screening of pregnant women [5].

In the clinical scenario, which is where this screening was done, a critical issue is the time needed to obtain reliable results from the cultures, which is 48 hours when using the methods recommended by CDC. Preterm labor and premature rupture of membranes are high-risk situations for early-onset sepsis, for which adequate intrapartum antibiotic prophylaxis is necessary. Rapid identification tests would be more suitable, since reliable and immediate results would be available, avoiding unnecessary antibiotics prescription. The only FDAapproved rapid test is a real-time PCR assay (IDI-Strep B ${ }^{\circledR}$ ), which has high sensitivity and specificity [15].

A study evaluating maternal colonization rates in 34 reports from developing countries found a GBS-prevalence rate of $12.7 \%$. However, when considering only those studies in which adequate laboratory methods were used (use of selective culture medium and collection of vaginal specimens), this rate increases to $17.8 \%$. Inappropriate microbiological methods were used on almost half of the patients included in this analysis [16].

The economic costs of universal maternal screening must be considered. We believe that given the enormous amount of money spent in intensive care units to care for infected newborns, and later to treat long-term disabilities caused by neonatal disease, the cost-benefit ratio favors culture screening. The Centers for Disease Control and Prevention estimated that $\$ 300$ million dollars were spent in a year to treat almost 7,500 cases of early-onset GBS disease [17]. There is no published evaluation of this issue in developing countries. In women with preterm labor, which had a prevalence of $25 \%$ 
Table 1. Clinical and obstetric characteristics and maternal colonization by group B Streptococcus

\begin{tabular}{|c|c|c|c|c|c|}
\hline Variable & $\begin{array}{c}\text { Colonized } \\
\mathbf{N}\end{array}$ & $\begin{array}{c}\text { Non colonized } \\
\mathbf{N}\end{array}$ & Total & $\begin{array}{c}\text { RR } \\
\text { (CI 95\%) }\end{array}$ & $\mathbf{P}$ \\
\hline \multicolumn{6}{|l|}{ Age } \\
\hline$<19$ & 12 & 26 & 38 & $1.7(0.7-2.02)$ & 0.541 \\
\hline$\geq 19$ & 44 & 121 & 165 & & \\
\hline \multicolumn{6}{|l|}{ Race } \\
\hline White & 36 & 69 & 105 & $1.65(1.08-2.69)$ & 0.027 \\
\hline Other & 20 & 78 & 98 & & \\
\hline \multicolumn{6}{|l|}{ Low level of education* } \\
\hline Yes & 37 & 73 & 110 & $1.70(1.03-2.81)$ & 0.031 \\
\hline No & 17 & 69 & 86 & & \\
\hline \multicolumn{6}{|l|}{ Gestational age } \\
\hline$<32$ weeks & 23 & 55 & 78 & $0.90(0.57-1.41)$ & 0.632 \\
\hline$\geq 32$ weeks & 33 & 92 & 125 & & \\
\hline \multicolumn{6}{|l|}{ Diagnosis } \\
\hline PPROM & 30 & 70 & 100 & $1.38(0.87-2.18)$ & 0.178 \\
\hline PTL & 26 & 77 & 103 & & \\
\hline \multicolumn{6}{|l|}{ Primigravid } \\
\hline Yes & 23 & 52 & 75 & $0.84(0.54-1.32)$ & 0.452 \\
\hline No & 33 & 95 & 128 & & \\
\hline Mean birth weight (g) & 2147 & 2361 & & $1.44(0.88-2.36)$ & 0.134 \\
\hline$\pm \mathrm{SD}$ & \pm 762 & \pm 744 & & & \\
\hline
\end{tabular}

$\mathrm{RR}=$ Risk Ratio $\mathrm{CI}=$ Confidence Interval; PPROM = Preterm premature rupture of membranes; PTL = Preterm labor; SD = Standard deviation; *less than elementary school completed.

Table 2. Group B Streptococcus detection results by type of culture medium

\begin{tabular}{lccc}
\hline & Positive & Negative & Total \\
\hline Non selective & $34(16.7 \%)$ & $169(83.3 \%)$ & $203(100 \%)$ \\
Selective & $49(24.1 \%)$ & $154(75.9 \%)$ & $203(100 \%)$ \\
Total & $83(20.4 \%)$ & $323(79.6 \%)$ & $406(100 \%)$ \\
\hline
\end{tabular}

GBS-positive mothers, initiating penicillin treatment before culture results would result in overtreatment of three out of four patients. This combined strategy of culturing women at risk while prescribing antibiotics has not been evaluated on a cost/benefit basis, which involves not only culture and antibiotic costs, but also pediatric care after birth. In our country, penicillin is a low-cost antibiotic.

We found that selective culture medium yielded the highest detection rates, with no difference between anorectal and vaginal samples. The proposed combined anorectal and vaginal swab directly inoculated into a tube containing Todd-Hewitt medium would cost about two U.S. dollars in Brazilian currency per patient. We believe that this low cost would be feasible even for universal screening at our maternity. This strategy was previously compared through delayed inoculation after sampling in transport media and gave better results, with enhanced detection rates [18].
Table 3. Group B Streptococcus culture results by anatomic site

\begin{tabular}{lcc}
\hline & Positive & Negative \\
\hline Vaginal & $45(22.2 \%)$ & $158(77.8 \%)$ \\
Anorectal & $45(22.2 \%)$ & $158(77.8 \%)$ \\
Total & $90(22.2 \%)$ & $316(77.8 \%)$ \\
\hline
\end{tabular}

\section{Conclusions}

The use of Todd-Hewitt selective medium yielded greater detection rates than non-selective medium. There was no difference in detection rates between vaginal and anorectal samples, however selective anorectal cultures detected more colonized women than the other three types of cultures.

From a practical standpoint, a single combined vaginalanorectal sample incubated in selective medium would be appropriate. This strategy would minimize laboratory costs without compromising maximum detection capacity, which is crucial for the prevention of early-onset neonatal disease.

\section{References}

1. Schuchat A., Zywicki S.S., Dinsmoor M.J., et al. Risk factors and opportunities for prevention of early-onset neonatal sepsis: a multicenter case-control study. Pediatrics 2000;105(Pt 1):21-6. 
2. Hyde T.B., Hilger T.M., Reingold A., et al. Active Bacterial Core surveillance (ABCs) of the Emerging Infections Program Network. Trends in incidence and antimicrobial resistance of early-onset sepsis: population-based surveillance in San Francisco and Atlanta. Pediatrics 2002;110:690-5.

3. Benitz W.E. Perinatal treatment to prevent early onset group B streptococcal sepsis. Semin Neonatol 2002;7(4):301-14.

4. Benitz W.E., Gould J.B., Druzin M.L. Risk factors for earlyonset group B streptococcal sepsis: estimation of odds ratios by critical literature review. Pediatrics 1999;103(6):77.

5. Schrag S., Gorwitz R., Fultz-Buts K., Schuchat A. Prevention of perinatal group $\mathrm{B}$ streptococcal disease. Revised guidelines from CDC. MMWR 2002;51(RR-11):1-22.

6. Mocelin C.O., Carvalho D.A.F., Brites C., et al. Isolamento de Streptococcus agalactiae de gestantes na regiäo de LondrinaPR. RBGO 1995 17:915-8.

7. Beraldo C., Brito A.S.J., Saridakis H.O., Matsuo T. Prevalência de colonização vaginal e anorretal por estreptococo do grupo B em gestantes no terceiro trimestre. RBGO 2004;26:543-9.

8. Philipson E.H., Palermino D.A., Robinson A. Enhanced antenatal detection of group B streptococcus colonization. Obstet Gynecol 1995;85:437-9.

9. Schuchat A. Group B streptococcal disease: from trials and tribulations to triumph and trepidation. Clin Infect Dis 2001;33(6):751-6.

10. Jaureguy F., Carton M., Teboul J., et al. Risk factors and screening strategy for group B streptococcal colonization in pregnant women: results of a prospective study. J Gynecol Obstet Biol Reprod 2003;32(2):132-8.
11. Madani T.A., Harding G.K., Helewa M., Alfa M.J. Screening pregnant women for group B streptococcal colonization. Infection. 1998;26(5):288-91.

12. Quinlan J.D., Hill D.A., Maxwell B.D., et al. The necessity of both anorectal and vaginal cultures for group B streptococcus screening during pregnancy. J Fam Pract 2000;49(5):447-8.

13. Platt M.W., McLaughlin J.C., Gilson G.J., et al. Increased recovery of group B Streptococcus by the inclusion of rectal culturing and enrichment. Diagn Microbiol Infect Dis 1995;21(2):65-8.

14. Gupta C., Briski L.E. Comparison of two culture media and three sampling techniques for sensitive and rapid screening of vaginal colonization by group B streptococcus in pregnant women. J Clin Microbiol 2004;42:3975-7.

15. Picard F.J., Bergeron M.G. Laboratory detection of group B Streptococcus for prevention of perinatal disease. Eur J Clin Microbiol Infect Dis 2004;23:665-71.

16. Stoll B.J., Schuchat A. Maternal carriage of group B streptococci in developing countries. Pediatr Infect Dis J 1998;17(6):499-503.

17. Centers for Disease Control and Prevention (CDC). Prevention of perinatal group B streptococcal disease: a public health perspective. MMWR Recomm Rep 1996;45(RR-7):1-24.

18. Silver H.M., Struminsky J. A comparison of the yield of positive antenatal group B Streptococcus cultures with direct inoculation in selective growth medium versus primary inoculation in transport medium followed by delayed inoculation in selective growth medium. Am J Obstet Gynecol 1996;175(1):155-7. 